\title{
Discectomia simples: ainda há espaço para a técnica?
}

\author{
Lumbar discectomy: is there still place for the technique? \\ Discectomía lumbar: todavía hay espacio para la técnica?
}

\author{
Felipe de Albuquerque Araujo Luyten' \\ René Kusabara ${ }^{2}$ \\ José Olympio Catão Bastos Júnior ${ }^{3}$ \\ Clóvis Yamazato ${ }^{3}$ \\ Fábio Mastromauro de Oliveira ${ }^{3}$ \\ Iberê Ribeiro ${ }^{3}$
}

\section{RESUMO}

Objetivo: avaliar retrospectivamente os resultados a longo prazo do procedimento de discectomia convencional como tratamento de dor ciática causada por hérnia discal lombar. Métodos: análise retrospectiva de 96 pacientes com hernia discal lombar, submetidos à discectomia convencional, com acompanhamento mínimo de dois anos. Resultados: pacientes apresentavam ciática esquerda em 54\% dos casos, com hérnia discal extrusa em $60 \%$ e 48\% no nível L4-5; média de idade de 42 anos, 53\% do sexo feminino, VAS médio de 9,6 no pré-operatório e 2,2 no pós-operatório. Conclusão: a discectomia convencional mostrou-se eficaz no tratamento da dor ciatica originada por hernia discal lombar, com baixo custo e restabelecimento precoce do paciente.

\section{ABSTRACT}

Objectives: retrospective evaluation of long term results of conventional discectomia as treatment to sciatic pain caused by lumbar disc hernia. Methods: retrospective analysis of 96 patients with lumbar disc hernia submitted to conventional discectomia, with minimum followup of 2 years. Results: the patients presented left sciatic pain in 54\% of the cases, with extruded herniation in $60 \%$ and $48 \%$ in the L4-5 level; mean age of 42 years, $53 \%$ female, medium VAN of 9.6 in preoperative and 2.2 in postoperative. Conclusion: the conventional discectomia was efficient in the treatment of sciatic pain originated by lumbar discal hernia, with low cost and fast recovery of the patient.

\section{RESUMEN}

Objetivos: evaluar de manera retrospectiva los resultados a largo plazo del procedimiento del discectomía convencional como tratamiento de dolor de la ciática causado por hernia discal lumbar. Métodos: análisis retrospectiva de 96 pacientes con hernia discal lumbar, que fueron sometidos al discectomía convencional, con el acompañamiento mínimo de dos años. Resultados: los pacientes presentaron ciática izquierda en el 54\% de los casos, con extrusa discal de la hernia en el $60 \%$ y el $48 \%$ en el nivel L4-5; una media de la edad de 42 años, el 53\% correspondiente al sexo femenino; el medio EAV de 9.6 en el preoperatorio y 2.2 en el postoperatorio. Conclusión: el discectomía convencional se reveló eficiente en el tratamiento del dolor de la ciática originado por hernia discal lumbar, con bajo costo y restablecimiento precoz del paciente.

DESCRIPTORES: Discectomía/ métodos; Desplazamiento del disco intervertebral; Procedimientos ortopédicos/ métodos; Ciática/cirugía; Resultado del tratamiento; Reoperación \footnotetext{
Campo (SP), Brasil; Hospital Geral de Guarulhos - Guarulhos (SP), Brasil.

'Estagiário do Grupo de Coluna do Hospital Municipal Cármino Caricchio - São Paulo (SP), Brasil.

${ }^{2}$ Chefe do Grupo de Coluna do Hospital Municipal Cármino Caricchio - São Paulo (SP), Brasil.

${ }^{3}$ Assistente do Grupo de Coluna do Hospital Municipal Cármino Caricchio - São Paulo (SP), Brasil.

Trabalho realizado no Hospital Municipal Cármino Caricchio; Hospital IGESP - São Paulo (SP), Brasil; Hospital IFOR Ortopedia e Traumatologia - São Bernardo do 


\section{INTRODUÇÃO}

A discectomia lombar é um procedimento cirúrgico comum, descrito por Mixter e Barr em 19341, com uma ampla margem de sucesso e satisfação (variando de 49 a 98 $\%$, dependendo dos critérios selecionados), conforme literatura analisada ${ }^{2-8}$.

A maioria desses trabalhos mostra que os resultados iniciais são bastante animadores ( $>90 \%$ ), porém evoluindo com uma queda ao longo do tempo. Deve-se salientar que as medidas usadas nesses estudos são critérios subjetivos, tais como satisfação relatada pelo paciente, dor residual e critérios de bom ou mau resultado estabelecidos pelo próprio cirurgião.

Apesar do aumento do uso do Short Form - 36 Health Survey (SF-36) como método de avaliação de resultados

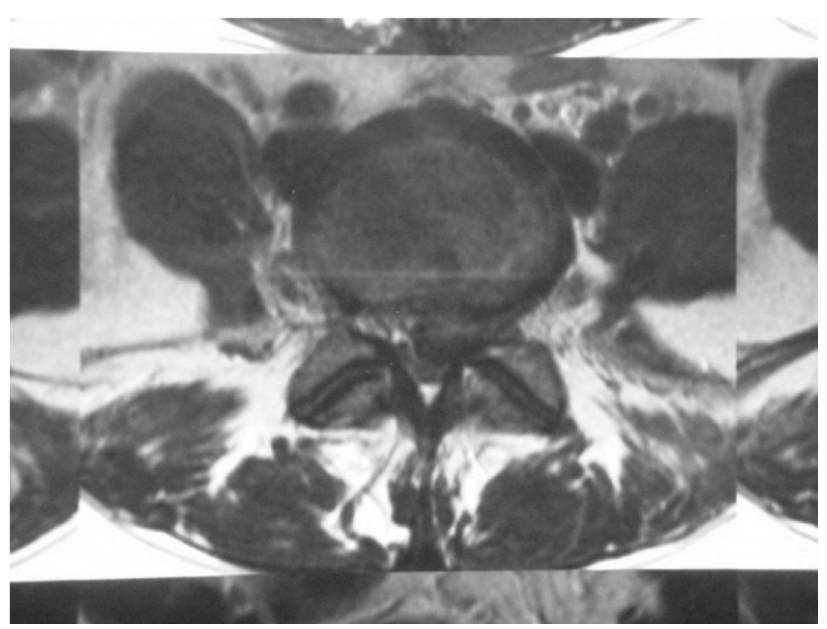

Figura 1

Corte axial de RM mostrando hérnia discal extrusa

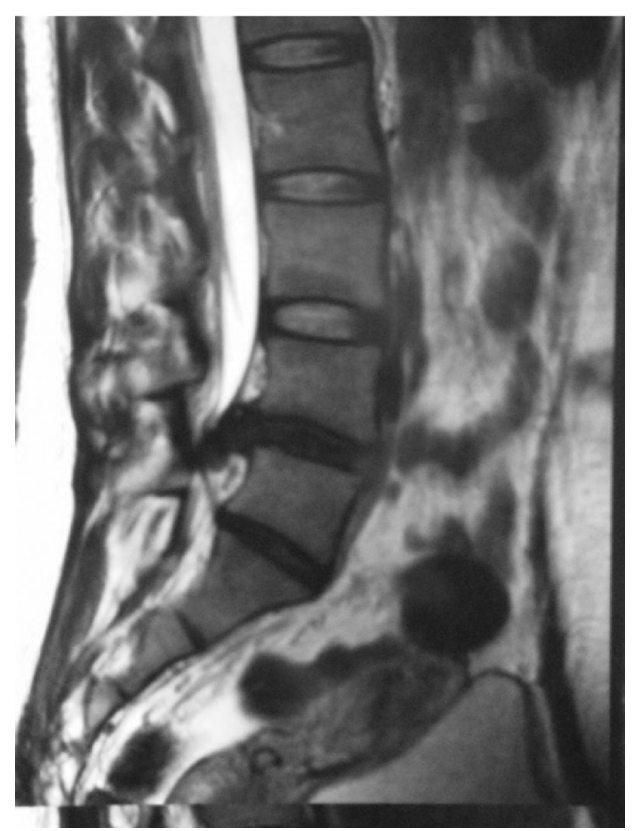

Figura 2

Corte sagital de RM mostrando hérnia discal extrusa funcionais, ainda existem poucas publicações com longos seguimentos que validem e confirmem essa ferramenta.

Recentemente, tem sido observado que a implantação de próteses, espaçadores e sistemas dinâmicos ou rígidos no tratamento das hérnias de disco da coluna lombar estão ganhando espaço no ambiente cirúrgico. Os autores fizeram um levantamento de pacientes operados pelo método de discectomia, sem colocação de nenhum tipo de implante, no período de dezembro de 1999 a março de 2007.

Este trabalho visa avaliar retrospectivamente os resultados em longo prazo do procedimento de discectomia convencional como tratamento de dor ciática, sendo que encontramos diversos estudos relacionados aos seus resultados em pacientes portadores de hérnia discal lombar.

\section{MÉTODOS}

Foram avaliados 96 pacientes com hérnia discal lombar, entre dezembro de 1999 e março de 2007, com acompanhamento mínimo de dois anos onde todos apresentavam dor do tipo ciática como sintoma principal. Todos os pacientes mantinha sintomas álgicos com achados clínicos compatíveis de radiculopatia e estavam sendo submetidos a tratamento conservador sem melhora por período superior a seis semanas. Todos os pacientes apresentavam exames de imagem (Figuras 1 a 4) evidenciando compressão radicular e foram operados pelo mesmo cirurgião.

Os pacientes foram submetidos à cirurgia utilizandose a técnica clássica descrita por $\mathrm{O}^{\prime} \mathrm{Conne}^{\circ}{ }^{9}$ (laminotomia com curetagem do espaço discal), em decúbito ventral, sob anestesia geral, com incisão mediana no nível da hérnia de cerca de cinco centímetros (variando apenas nos pacientes obesos), com técnica de hemi-hemi laminectomia na vértebra proximal, ressecando-se o ligamento amarelo. Após afastamento medial da raiz nervosa, ressecava-se o fragmento extruso quando havia lesão prévia do ligamento longitudinal posterior. Quando não, incisava-se o ligamento, ressecando-se a hérnia discal, com limpeza do núcleo pulposo com cureta e pinça de disco. Era colocada uma

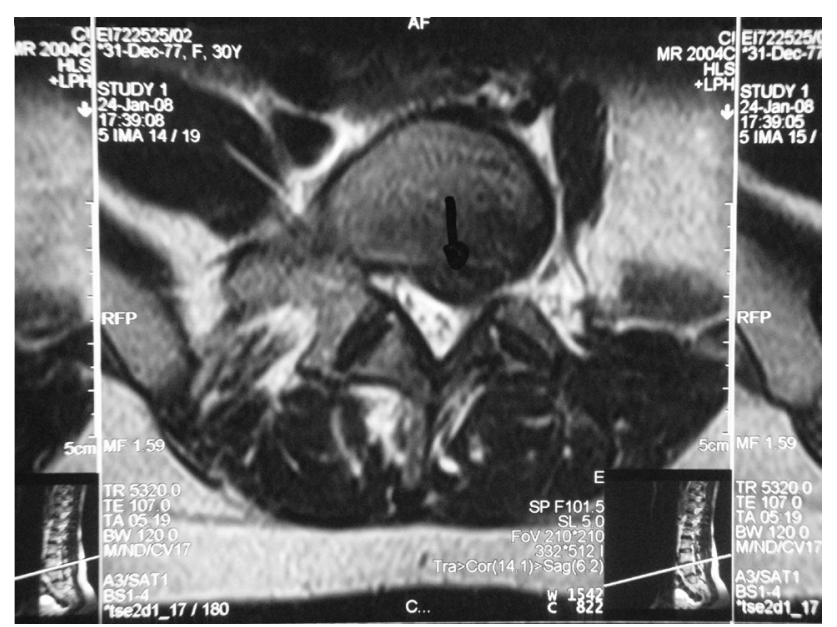

Figura 3

Corte axial de RM mostrando hérnia discal lateral 


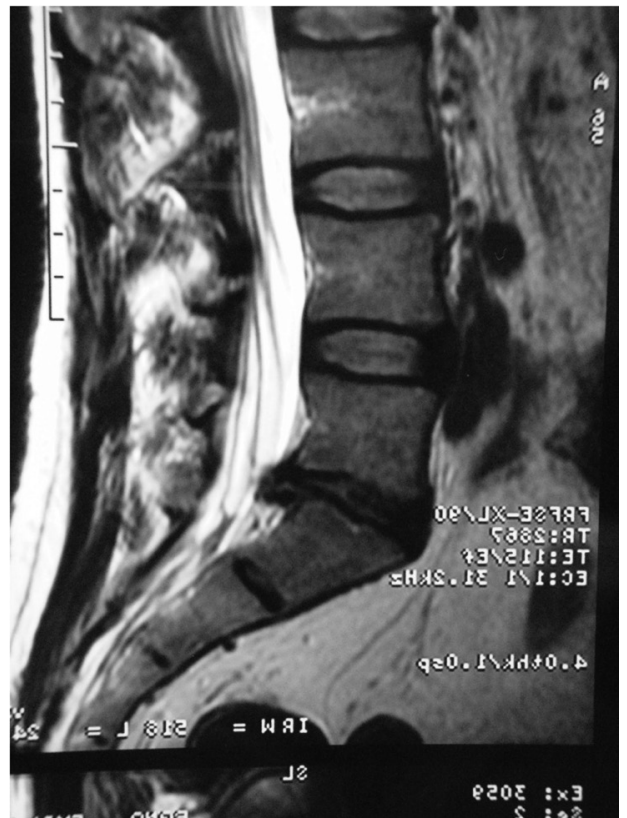

Figura 4

Corte sagital de RM mostrando hérnia discal lateral

pequena camada de proteção com esponja hemostática absorvível, sutura da aponeurose com fio inabsorvível, tecido celular subcutâneo e pele com fios absorvíveis, infiltrandose o tecido celular subcutâneo com $10 \mathrm{~mL}$ de bupivacaína. Os mesmos eram encorajados a andar no pós-operatório imediato, recebendo analgesia endovenosa até a alta, variando entre um e oito dias, com 2,4 dias em média. A antibioticoterapia profilática era aplicada apenas na indução anestésica, com um grama de cefazolina.

\section{RESULTADOS}

Em relação ao tipo da hérnia, 58 pacientes $(60 \%)$ apresentavam hérnia discal extrusa, com lesão do ligamento longitudinal posterior. Em 28 pacientes, a hérnia era lateral $(29 \%)$, em seis era central (6\%) e em quatro era centrolateral (4\%).

Os sintomas de ciática foram observados à esquerda em 54 pacientes $(56 \%)$ e à direita em $42(44 \%)$, determinando a abordagem cirúrgica, efetuada do lado mais sintomático.

Com relação à localização, 46 pacientes apresentavam hérnia no nível L4-L5 (48\%), 41 em L5-S1 (43\%), 4 em L3-L4 (4\%), 2 em L2-L3 (2\%) e 3 apresentavam hérnias em dois níveis, ambas operadas.

A idade variou entre 17 e 81 anos, com média de 42 anos; 51 pacientes eram do sexo feminino (53\%) e 45 do sexo masculino (47\%).

Os pacientes foram avaliados pela escala visual analógica (EVA), no pré-operatório e no pós-operatório imediato, após duas semanas, quando eram retirados os pontos e com seis semanas, três meses, seis meses, um e dois anos.

A média foi de 9,6 no pré-operatório e de 2,2 no pósoperatório imediato. Passando a 1,8 na retirada de pontos, seis semanas após a cirurgia passou a 1,7; três meses após passou a 1,7; seis meses após passou a 1,9; um ano após passou a 1,9 e dois anos após passou a 1,8. Apenas 17 pacientes foram avaliados após os 2 anos, dos quais alguns retornaram por nova crise dolorosa e outros por terem sido submetidos a artrodese posteriormente.

$\mathrm{O}$ retorno às atividades físicas era encorajado a partir de três meses. Não foi possível avaliar o retorno ao trabalho, pois a maioria dos pacientes permaneceu afastada durante mais tempo por motivos pessoais e sociais.

As complicações foram diversas, em que sete pacientes (7\%) apresentaram parestesia pós-operatória e apenas um apresentou déficit motor (1\%), todos com regressão total. Quatro tiveram infecção superficial (4\%). Uma paciente evoluiu com infecção urinária (1\%). Dois pacientes sofreram lesão da dura-máter no intraoperatório (2\%), dos quais um permaneceu internado por quatro dias, sem sintomas de fístula liquórica. $\mathrm{O}$ outro paciente evoluiu com quadro de discite, necessitando de antibioticoterapia oral por três meses, tendo sido submetido à cirurgia por estenose do canal após três anos.

Além desse paciente, outros 14 foram submetidos à nova cirurgia por recidiva da hérnia discal $(15 \%)$, e um foi submetido a nova discectomia (1\%). Os outros 13 foram submetidos à artrodese, com emprego de parafusos pediculares. Dois outros pacientes apresentaram recidiva da hérnia, com regressão dos sintomas por meio de tratamento clínico.

\section{DISCUSSÃO}

O tratamento para a doença degenerativa da coluna lombar é bastante controverso, principalmente quando existe estreitamento do canal ou instabilidade.

Os autores fizeram esse levantamento de pacientes com hérnia discal sintomática - a maioria com fragmento extruso em que o principal sintoma era ciatalgia do lado acometido pela hérnia.

A grande maioria apresentou remissão quase total do sintoma doloroso, com incisão mínima e internação curta, levando a rápida recuperação para retorno às atividades laborais $^{2-8}$. O uso da infiltração de anestésico (bupivacaína) no tecido celular subcutâneo e musculatura adjacente na região cirúrgica auxiliou na analgesia pós-operatoria inicial ${ }^{10}$.

$\mathrm{O}$ custo também foi baixo quando comparado à literatura internacional ${ }^{11}$. Como não há inclusão de material especial de osteossíntese, uma internação para esse tipo de cirurgia (duas diárias) apresenta valores entre $\mathrm{R} \$ 3.300,00$ e R \$ 8.000,00 em alguns hospitais pesquisados na região central de São Paulo.

O número de pacientes que necessitaram de reoperação por recidiva da hérnia foi de 14 (15\%), semelhante à maioria dos trabalhos estudados ${ }^{4-8}$, sendo que um paciente foi submetido a reoperação, pois não queria ser submetido a artrodese, enquanto que os outros foram submetidos a artrodese lombar, variando de três meses a três anos após a discectomia. Dois pacientes ainda apresentaram recidiva da hérnia, melhorando os sintomas com tratamento clínico. 
Apesar de a casuística apresentar discopatia adjacente em três pacientes (hérnia em L4-5 e discopatia em L5-S1) e instabilidade adjacente em seis, apenas um (1\%) evoluiu com recidiva da hérnia, necessitando de artrodese. Todos os outros evoluíram com boa melhora dos sintomas após a discectomia.

Os pacientes não eram encorajados à realização de fisioterapia pós-operatória, e 33 (34\%) não realizaram tal tratamento. Quando o paciente apresentava sintomas dolorosos ou neurológicos, era indicada a fisioterapia, que, em média, foi iniciada após quatro semanas, mantendo-se até remissão dos sintomas, semelhante à literatura internacional $^{12}$, com retorno às funções por volta de três a quatro meses, em média.
Dois pacientes sofreram lesão dural (2\%), o que se assemelha com a literatura internacional ${ }^{13}$, variando de 2 a $14 \%$ em discectomias simples.

Mesmo aqueles pacientes com hérnia discal em dois níveis ou com hérnia central obtiveram bom resultado, pois todos eles apresentavam dor ciática como sintoma principal.

\section{CONCLUSÃO}

A técnica de discectomia simples como tratamento cirúrgico da hérnia discal lombar é eficiente, de baixo custo, levando a restabelecimento precoce do paciente. Os pacientes que apresentaram hérnia extrusa obtiveram melhor resultado quanto ao alívio da dor ciática, sem sinais de instabilidade ou estenose do canal no nível da hérnia.

\section{REFERÊNCIAS}

1. Mixter WJ, Barr JS. Rupture of the intervertebral disc with involvement of the spinal canal. N Engl J Med. 1934;211:210-5.

2. Davis RA. A long-term outcome analysis of 984 surgically treated herniated lumbar discs. J Neurosurg. 1994;80(3):415-21.

3. Dvorak J, Gauchat MH, Valach L. The outcome of surgery for lumbar disc herniation. I. A 4-17 years' follow up with emphasis on somatic aspects. Spine (Phila Pa 1976). 1988;13(12):1418-22.

4. Morgan-Hough CV, Jones PW, Eisenstein SM. Primary and revision lumbar discectomy. A 16-year review from one centre. J Bone Joint Surg Br. 2003;85(6):871-4.

5. Loupasis GA, Stamos K, Katonis PG, Sapkas G, Korres DS, Hartofilakidis G. Seven- to 20-year outcome of lumbar discectomy. Spine (Phila Pa 1976). 1999; 24: 2313-7.

6. Yorimitsu E, Chiba K, Toyama Y, Hirabayashi K. Long-term outcomes of standard discectomy for lumbar disc herniation: a follow-up study of more than 10 years. Spine (Phila Pa 1976). 200;26(6):652-7.
7. Mariconda M, Galasso O, Secondulfo V, Rotonda GD, Milano C. Minimum 25-year outcome and functional assessment of lumbar discectomy. Spine (Phila Pa 1976). 2006;31(22):2593-9.

8. Carragee EJ, Han MY, Suen PW, Kim D. Clinical outcomes after lumbar discectomy for sciatica: the effects of fragment type and anular competence. J Bone Joint Surg Am. 2003;85$\mathrm{A}(1): 102-8$.

9. O'Connell JE. Protrusions of the lumbar intervertebral discs, a clinical review based on five hundred cases treated by excision of the protrusion. J Bone Joint Surg Br. 1951;33$\mathrm{B}(1): 8-30$

10. Ersayli DT, Gurbet A, Bekar A, Uckunkaya N, Bilgin H. Effects of perioperatively administered bupivacaine and bupivacainemethylprednisolone on pain after lumbar discectomy. Spine (Phila Pa 1976). 2006;31(19):2221-6.

11. Malter AD, Larson EB, Urban N, Deyo RA. Cost-effectiveness of lumbar discectomy for the treatment of herniated intervertebral disc. Spine (Phila Pa 1976). 1996;21(9):1048-54.
12. Carragee EJ, Han MY, Yang B, Kim DH, Kraemer H, Billys J. Activity restrictions after posterior lumbar discectomy. A prospective study of outcomes in 152 cases with no postoperative restrictions. Spine (Phila Pa 1976). 1999;24(22):2346-51.

13. Yurac R, Marre B, Rojas C, Ramirez S, Munjin M, Urzua A, Lecaros MA, Ilabaca F, Fleiderman J. Durotomias incidentales en discectomias lumbares. Coluna. 2008;7(4):371-5.

\section{Correspondência}

Felipe de Albuquerque Araujo Luyten

Rua da Consolação, 222, conj. 301 Centro

CEP: 01302-000 - São Paulo (SP), Brasil

Tel: (1 1 ) 3256-6799

E-mail: felipe_luyten@yahoo.com 